PROCEEDINGS OF THE

AMERICAN MATHEMATICAL SOCIETY

Volume 136, Number 11, November 2008, Pages 4089-4093

S 0002-9939(08)09390-8

Article electronically published on June 4, 2008

\title{
A SUFFICIENT AND NECESSARY CONDITION FOR THE CONVERGENCE OF THE SEQUENCE OF SUCCESSIVE APPROXIMATIONS TO A UNIQUE FIXED POINT
}

\author{
TOMONARI SUZUKI
}

(Communicated by N. Tomczak-Jaegermann)

\begin{abstract}
If $(X, d)$ is a complete metric space and $T: X \rightarrow X$ is a contraction mapping, then the conclusion of the Banach-Caccioppoli contraction principle is that the sequence of successive approximations of $T$ starting from any point of the space converges to a unique fixed point. In this paper, we obtain a sufficient and necessary condition of the above conclusion in terms of the so-called strong Leader mappings.
\end{abstract}

\section{INTRODUCTION}

The following famous theorem is referred to as the Banach-Caccioppoli contraction principle. This theorem is very forceful and simple, and it became a classical tool in nonlinear analysis.

Theorem 1 (Banach [1] and Caccioppoli [2]). Let $(X, d)$ be a complete metric space and let $T$ be a contraction mapping on $X$; i.e., there exists $r \in[0,1)$ such that $d(T x, T y) \leq r d(x, y)$ for all $x, y \in X$. Then the following holds:

(A) $T$ has a unique fixed point, and for any $x \in X$ the sequence of successive approximations $\left\{T^{n} x\right\}$ converges to the fixed point.

There are thousands of fixed point theorems in metric spaces. However, the conclusion of most of theorems is (A). For example, the conclusion of Ćirić's [5], Edelstein's [6], Kannan's 8], Kirk's [10, Matkowski's [12, Meir-Keeler's [13] and Suzuki's [15, 16] fixed point theorems is (A), There are a few exceptions. For example, the conclusions of Caristi's [3, 4] and Subrahmanyam's 14] fixed point theorems and their generalizations are not (A). See Kirk's survey 9 .

In 1983, Leader obtained a sufficient and necessary condition of some condition which is closely connected with $(\mathrm{A})$, See also [7, 18.

Definition 1 (Leader [1]). Let $T$ be a mapping on a metric space $(X, d)$. Then $T$ is called a Leader mapping if the following holds:

Received by the editors August 20, 2007, and, in revised form, October 12, 2007.

2000 Mathematics Subject Classification. Primary 54H25.

Key words and phrases. Fixed point, successive approximations, Banach-Caccioppoli contraction principle, Leader mapping.

The author was supported in part by Grants-in-Aid for Scientific Research from the Japanese Ministry of Education, Culture, Sports, Science and Technology.

(C)2008 American Mathematical Society Reverts to public domain 28 years from publication 
(B) For $x, y \in X$ and $\varepsilon>0$, there exist $\delta>0$ and $\nu \in \mathbb{N}$ such that

$$
d\left(T^{i} x, T^{j} y\right)<\varepsilon+\delta \quad \text { implies } \quad d\left(T^{i+\nu} x, T^{j+\nu} y\right)<\varepsilon
$$

for all $i, j \in \mathbb{N} \cup\{0\}$, where $T^{0}$ is the identity mapping on $X$.

Theorem 2 (Leader [11]). Let $T$ be a mapping on a metric space $(X, d)$. Then $T$ is a Leader mapping if and only if the following holds:

(C) $\left\{T^{n} x\right\}$ is a Cauchy sequence and $\lim _{n} d\left(T^{n} x, T^{n} y\right)=0$ for all $x, y \in X$.

Conditions (A) and (C) are very close. However, Theorem 2 does not assure the existence of fixed points. So from this point of view, we might be able to consider that both conditions are quite different. The author thinks that Theorem 2 is excellent; however, it is not perfect. In this paper, we proceed with Leader's study and complete the study. That is, we obtain a sufficient and necessary condition of $(\mathrm{A})$.

\section{MAIN RESULTS}

Throughout this paper we denote by $\mathbb{N}$ the set of all positive integers.

Since Condition $(\mathrm{C})$ is weaker than $(\mathrm{A})$, we have to introduce a condition which is stronger than (B)

Definition 2. Let $T$ be a mapping on a metric space $(X, d)$. Then $T$ is called a strong Leader mapping if (B) and the following hold:

(D) For $x, y \in X$, there exist $\nu \in \mathbb{N}$ and a sequence $\left\{\alpha_{n}\right\}$ in $(0, \infty)$ such that

$$
d\left(T^{i} x, T^{j} y\right)<\alpha_{n} \quad \text { implies } \quad d\left(T^{i+\nu} x, T^{j+\nu} y\right)<1 / n
$$

for all $i, j \in \mathbb{N} \cup\{0\}$ and $n \in \mathbb{N}$.

We shall prove that Condition (A) and the conjunction of Conditions (B) and (D) are equivalent.

Theorem 3. Let $(X, d)$ be a complete metric space and let $T$ be a strong Leader mapping on $X$. Then (A) holds.

Proof. By Theorem 2, $\left\{T^{n} x\right\}$ is a Cauchy sequence and $\lim _{n} d\left(T^{n} x, T^{n} y\right)=0$ for all $x, y \in X$. Since $X$ is complete, there exists $z \in X$ such that $\lim _{n} T^{n} x=z$ for all $x \in X$. From this, there exists at most one fixed point of $T$. Fix $x \in X$. From (D), there exist $\nu \in \mathbb{N}$ and a sequence $\left\{\alpha_{n}\right\}$ in $(0, \infty)$ such that

$$
d\left(T^{i} x, T^{j} z\right)<\alpha_{n} \quad \text { implies } \quad d\left(T^{i+\nu} x, T^{j+\nu} z\right)<1 / n
$$

for all $i, j \in \mathbb{N} \cup\{0\}$ and $n \in \mathbb{N}$. For $k \in \mathbb{N}$, there exists $\ell \in \mathbb{N}$ such that $\ell \geq k$ and $d\left(T^{\ell} x, z\right)<\alpha_{k}$ because $\lim _{n} T^{n} x=z$. So we have

$$
d\left(T^{\ell+\nu} x, T^{\nu} z\right)<1 / k .
$$

As $k$ tends to $\infty$, we obtain $d\left(z, T^{\nu} z\right)=0$; that is, $T^{\nu} z=z$. Since $\lim _{n} T^{n} z=z, z$ is a fixed point of $T$.

Theorem 4. Let $(X, d)$ be a metric space and let $T$ be a mapping on $X$. Assume that (A) holds. Then $T$ is a strong Leader mapping. 
Proof. Let $z \in X$ be a unique fixed point of $T$. From the assumption, $\left\{T^{n} x\right\}$ is a Cauchy sequence and $\lim _{n} d\left(T^{n} x, T^{n} y\right)=d(z, z)=0$ for all $x, y \in X$. So by Theorem 2. $T$ satisfies (B). Let us prove (D), Fix $x, y \in X$ and $n \in \mathbb{N}$. We choose $L \in \mathbb{N}$ such that $d\left(T^{m} x, z\right)<1 /(2 n)$ and $d\left(T^{m} y, z\right)<1 /(2 n)$ for all $m \in \mathbb{N}$ with $m>L$. Put

$$
\begin{aligned}
\alpha_{n}:=\min \{\min & \left\{\inf \left\{d\left(T^{\ell} x, T^{m} y\right): m \in \mathbb{N} \cup\{0\}, T^{m} y \neq T^{\ell} x\right\}\right. \\
& \left.: \ell \in\{0,1, \cdots, L\}, T^{\ell} x \neq z\right\}, \\
\min \{ & \inf \left\{d\left(T^{\ell} y, T^{m} x\right): m \in \mathbb{N} \cup\{0\}, T^{m} x \neq T^{\ell} y\right\} \\
& \left.: \ell \in\{0,1, \cdots, L\}, T^{\ell} y \neq z\right\}, \\
1\}, &
\end{aligned}
$$

where $\min \varnothing=\infty$ and $\inf \varnothing=\infty$.

We shall show $\alpha_{n}>0$. If not, then without loss of generality, we may assume there exists $\lambda \in\{0,1, \cdots, L\}$ such that

$$
T^{\lambda} x \neq z \quad \text { and } \quad \inf \left\{d\left(T^{\lambda} x, T^{m} y\right): m \in \mathbb{N} \cup\{0\}, T^{m} y \neq T^{\lambda} x\right\}=0 .
$$

Then we can choose a sequence $\left\{m_{j}\right\}$ in $\mathbb{N} \cup\{0\}$ such that

$$
T^{m_{j}} y \neq T^{\lambda} x \quad \text { and } \quad \lim _{j \rightarrow \infty} d\left(T^{\lambda} x, T^{m_{j}} y\right)=0 .
$$

Since $T^{m_{j}} y \neq T^{\lambda} x$ for all $j \in \mathbb{N}$, we have $\lim _{j} m_{j}=\infty$ and hence

$$
d\left(T^{\lambda} x, z\right)=\lim _{j \rightarrow \infty} d\left(T^{\lambda} x, T^{m_{j}} y\right)=0 .
$$

This is a contradiction. Therefore we obtain $\alpha_{n}>0$.

Fix $i, j \in \mathbb{N} \cup\{0\}$ with $d\left(T^{i} x, T^{j} y\right)<\alpha_{n}$. We consider the following cases:

(i) $T^{i} x=T^{j} y$,

(ii) $i \leq L$ and $T^{i} x \neq z$,

(iii) $j \leq L$ and $T^{j} y \neq z$,

(iv) $T^{i} x=z$ and $T^{j} y=z$,

(v) $T^{i} x=z$ and $j>L$,

(vi) $T^{j} y=z$ and $i>L$,

(vii) $i>L$ and $j>L$.

\begin{tabular}{c||c|c|c} 
& $i \leq L, T^{i} x \neq z$ & $i \leq L, T^{i} x=z$ & $i>L$ \\
\hline \hline$j \leq L, T^{j} y \neq z$ & (ii), (iii) & (iii) & (iii) \\
\hline$j \leq L, T^{j} y=z$ & (ii) & (iv) & (vi) \\
\hline$j>L$ & (ii) & (v) & (vii)
\end{tabular}

In the first case, it is obvious that $d\left(T^{i+1} x, T^{j+1} y\right)=0<1 / n$. In the second case, we have

$$
d\left(T^{i} x, T^{j} y\right)<\alpha_{n} \leq \inf \left\{d\left(T^{i} x, T^{m} y\right): m \in \mathbb{N} \cup\{0\}, T^{m} y \neq T^{i} x\right\}
$$

and hence $T^{i} x=T^{j} y$. Thus the first case includes the second case. Similarly the first case includes the third case. Also, it is obvious that the first case includes the fourth case. In the fifth case, we have

$$
d\left(T^{i+1} x, T^{j+1} y\right)=d\left(T z, T^{j+1} y\right)=d\left(z, T^{j+1} y\right)<1 /(2 n)<1 / n .
$$


In the sixth case, we can prove $d\left(T^{i+1} x, T^{j+1} y\right)<1 / n$ as in the fifth case. In the seventh case, we obtain

$$
d\left(T^{i+1} x, T^{j+1} y\right) \leq d\left(T^{i+1} x, z\right)+d\left(T^{j+1} y, z\right)<1 /(2 n)+1 /(2 n)=1 / n .
$$

Thus we obtain (D)

From the proof Theorem 4 , we obtain the following.

Theorem 5. Let $T$ be a mapping on a complete metric space $(X, d)$. Then the following are equivalent:

(i) (A) holds.

(ii) $T$ is a strong Leader mapping.

(iii) (B) and the following hold:

(E) For $x, y \in X$, there exists a sequence $\left\{\alpha_{n}\right\}$ in $(0, \infty)$ such that

$$
d\left(T^{i} x, T^{j} y\right)<\alpha_{n} \quad \text { implies } \quad d\left(T^{i+1} x, T^{j+1} y\right)<1 / n
$$

for all $i, j \in \mathbb{N} \cup\{0\}$ and $n \in \mathbb{N}$.

\section{Additional Result}

We finally give an alternative proof of Lemma 2 in [11] (Lemma 11 below). The lemma played a very important role in the proof of Theorem 2 Since Leader proved Lemma 1 by reductio ad absurdum, we will give a proof without reductio ad absurdum. See also the proof of Theorem 1 in [17.

Lemma 1 (Leader [11]). Let $\left\{x_{n}\right\}$ and $\left\{y_{n}\right\}$ be sequences in a metric space $(X, d)$. Let $\varepsilon \in(0, \infty), \delta \in(0, \infty]$ and $\nu \in \mathbb{N}$ satisfy

$$
d\left(x_{i}, y_{j}\right)<\varepsilon+\delta \quad \text { implies } \quad d\left(x_{i+\nu}, y_{j+\nu}\right)<\varepsilon
$$

for all $i, j \in \mathbb{N}$. Let $n \in \mathbb{N}$ satisfy

$$
\max \left\{d\left(x_{i}, y_{j}\right): i, j \in\{n, n+1, \cdots, n+\nu\}\right\}<\min \{\varepsilon, \delta / 2\} .
$$

Then $d\left(x_{i}, y_{j}\right)<3 \varepsilon$ for all $i, j \in \mathbb{N}$ with $i \geq n$ and $j \geq n$.

Proof. We first show by induction

$$
d\left(x_{n+\nu}, y_{n+m}\right)<\varepsilon
$$

for all $m \in \mathbb{N} \cup\{0\}$. From the assumption, (1) holds for $m=0,1, \cdots, \nu$. We assume (11) holds for some $m \in \mathbb{N}$. That is, $d\left(x_{n+\nu}, y_{n+m}\right)<\varepsilon$, which implies

$$
d\left(x_{n}, y_{n+m}\right) \leq d\left(x_{n}, y_{n}\right)+d\left(y_{n}, x_{n+\nu}\right)+d\left(x_{n+\nu}, y_{n+m}\right)<\delta / 2+\delta / 2+\varepsilon=\varepsilon+\delta .
$$

From the assumption, we have $d\left(x_{n+\nu}, y_{n+m+\nu}\right)<\varepsilon$. Thus, (1) holds when $m:=$ $m+\nu$. By induction, (11) holds for all $m \in \mathbb{N} \cup\{0\}$. Similarly we can prove $d\left(y_{n+\nu}, x_{n+m}\right)<\varepsilon$ for all $m \in \mathbb{N} \cup\{0\}$. Therefore we obtain

$$
d\left(x_{i}, y_{j}\right) \leq d\left(x_{i}, y_{n+\nu}\right)+d\left(y_{n+\nu}, x_{n+\nu}\right)+d\left(x_{n+\nu}, y_{j}\right)<\varepsilon+\varepsilon+\varepsilon=3 \varepsilon
$$

for $i, j \in \mathbb{N}$ with $i \geq n$ and $j \geq n$.

\section{ACKNOWLEDGEMENT}

The author is very grateful to the referee for suggestions that improved the presentation of this paper. 


\section{REFERENCES}

[1] S. Banach, Sur les opérations dans les ensembles abstraits et leur application aux équations intégrales, Fund. Math., 3 (1922), 133-181.

[2] R. Caccioppoli, Un teorema generale sull'esistenza di elementi uniti in una transformazione funzionale, Rend. Accad. Naz. Lincei, 11 (1930), 794-799.

[3] J. Caristi, Fixed point theorems for mappings satisfying inwardness conditions, Trans. Amer. Math. Soc., 215 (1976), 241-251. MR[0394329 (52:15132)

[4] J. Caristi and W. A. Kirk, Geometric fixed point theory and inwardness conditions, Lecture Notes in Math., Vol. 490, pp. 74-83, Springer, Berlin, 1975. MR0399968 (53:3806)

[5] Lj. B. Ćirić, A generalization of Banach's contraction principle, Proc. Amer. Math. Soc., 45 (1974), 267-273. MR0356011 (50:8484)

[6] M. Edelstein, On fixed and periodic points under contractive mappings, J. London Math. Soc., 37 (1962), 74-79. MR0133102 (24:A2936)

[7] J. Jachymski, An iff fixed point criterion for continuous self-mappings on a complete metric space, Aequationes Math., 48 (1994), 163-170. MR.1295089 (96c:54069)

[8] R. Kannan, Some results on fixed points - II, Amer. Math. Monthly, 76 (1969), 405-408. MR0257838 (41:2487)

[9] W. A. Kirk, Contraction mappings and extensions, in Handbook of Metric Fixed Point Theory (W. A. Kirk and B. Sims, Eds.), 2001, pp. 1-34, Kluwer Academic Publishers, Dordrecht. MR1904272 (2003f:54096)

[10] , Fixed points of asymptotic contractions, J. Math. Anal. Appl., 277 (2003), 645-650. MR.1961251 (2003k:47093)

[11] S. Leader, Equivalent Cauchy sequences and contractive fixed points in metric spaces, Studia Math., 76 (1983), 63-67. MR728197 (85c:54081)

[12] J. Matkowski, Integrable solutions of functional equations, Diss. Math., 127, Warsaw, 1975. MR0412650 (54:772)

[13] A. Meir and E. Keeler, A theorem on contraction mappings, J. Math. Anal. Appl., 28 (1969), 326-329. MR0250291(40:3530)

[14] P. V. Subrahmanyam, Remarks on some fixed point theorems related to Banach's contraction principle, J. Math. Phys. Sci., 8 (1974), 445-457. MR0358749 (50:11208)

[15] T. Suzuki, Generalized distance and existence theorems in complete metric spaces, J. Math. Anal. Appl., 253 (2001), 440-458. MR1808147(2002f:49038)

[16] - Several fixed point theorems concerning $\tau$-distance, Fixed Point Theory Appl., 2004, no. 3, 195-209. MR.2096951

[17] Some notes on Meir-Keeler contractions and L-functions, Bull. Kyushu Inst. Technol., 53 (2006), 1-13. MR2237618 (2007g:54068)

[18] _ A definitive result on asymptotic contractions, J. Math. Anal. Appl., 335 (2007), 707-715. MR2340349

Department of Mathematics, Kyushu Institute of Technology, Sensuicho, Tobata, KITAKYUSHU 804-8550, JAPAN

E-mail address: suzuki-t@mns.kyutech.ac.jp 